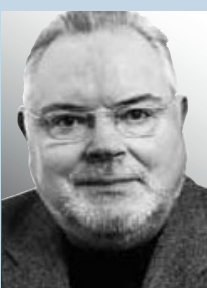

Dr. med.

Dieter Leithäuser

HNO-Arzt, Warburg

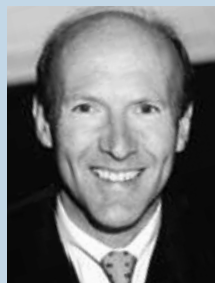

Prof. Dr. med.

Ralph Mösges

Universität Köln die Studien auch nahelegen: nur erheblich kranke Kinder behandeln; den Eltern die Entscheidung mit „Bedarfsrezept“ überlassen)? Soll man erst warten (wait-and see für 48 Stunden, dann die Spontanerholer-Versager behandeln)? Wie lange soll man behandeln (Cochrane 2010: 5 Tage genügen, 10 sind besser), oder bis oder ab welchem Alter therapieren (die Studien umfassten nur Kinder im Alter von 6 Monaten bis 3 Jahren)? Also besteht noch viel Spielraum für weitere Diskussionen und nichts ist entschieden.

Einige interessante Nebenaspekte der Studien sollen nicht unerwähnt bleiben: In der Studie von Tähtinen et al. (die mit der geringeren Antibiotika-Dosis) waren explizit abschwellende Nasentropfen und Sprays zugelassen, die ja für sich genommen auch als Behandlung gelten. In der Studie von Hoberman et al gab es eine Mastoiditis in der Plazebo-Gruppe, und in beiden Studien wur- den deutlich mehr Trommelfellperforationen und Rezidive in den Plazebo-Gruppen beobachtet. Honi soit qui mal y pense?

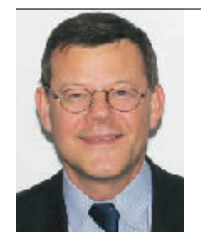

Prof Dr. med. Olaf Michel Universitair Ziekenhuis, Brüssel

\section{Hörverlust nach Strahlentherapie von Kopf- und Halskarzinomen}

Die Behandlung von Kopf- und Halstumoren schließt oft Methoden ein, die das Hörsystem beeinflussen und einen Hörverlust verursachen. Die Bestrahlung der Nackenregion stört die Lymphdrainage, die Chemotherapie mit zytotoxischen Medikamenten ist auch toxisch für das Ohr und kann zu Hörverlusten verschiedenen Typs und Grades führen.

W issenschaftler eines Regelkrankenhauses in brasilianischen São Paulo untersuchten in einer prospektiven Fall-Kontroll-Studie 282 Teilnehmer, davon 141 mit Hals- und Kopftumoren, die sich einer Strahlentherapie unterzogen hatten und diese seit mindestens zwei Jahren rezidivfrei überlebt hatten. Die 141 Probanden einer altersentsprechenden Kontrollgruppe waren wegen Tumoren außerhalb des HNO-Bereiches ausschließlich chirurgisch behandelt worden war.

Alle Personen wurden mit verschiedenen Methoden audiometrisch evaluiert: Die Strahlendosis, die auf das Hörsystem einwirkte, wurde als Prozentanteil des äußeren Strahlenfeldes angegeben.
Ein größerer Hörverlust wurde bei 102 der Teilnehmer (72,3\%) mit Strahlentherapie festgestellt. In der Kontrollgruppe war trat ein Hörverlust nur bei 69 Teilnehmern $(48,9 \%)$ auf ( $\mathrm{p}<0,001)$. Beim Hörverlust in der Kontrollgruppe handelte es sich meist um eine milde Schallempfindungsschwerhörigkeit. Die Probanden der Strahlentherapiegruppe hatten hingegen häufiger schwerere Formen des Hörverlusts und gemischte Hörverlusttypen.

Eine Anakusis lag rechts zu 5,4\% und links zu 8,4\% vor versus $1,4 \%$ bei der Kontrollgruppe. 19,1\% der Probanden der Strahlentherapiegruppe waren durch den Hörverlust schwer behindert ( $\mathrm{p}<$ 0,001 ), in der Kontrollgruppe galt dies nur für $2,8 \%$.

K. Malberg

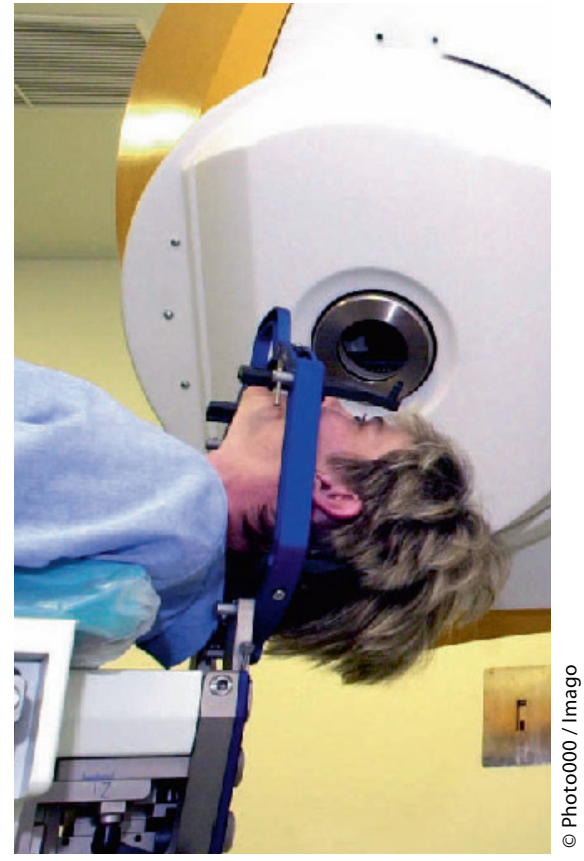

Das Hörsystem wird mitbestrahlt.

Schultz $\mathrm{C}$ et al. Hearing loss and complaint in patients with head and neck cancer treated with radiotherapy. Arch Otolaryngol Head Neck Surg. 136; 2010: 1065-9 